\title{
Emerging Dynamic Design Techniques for Mechanical and Structural Systems
}

\section{Foreword}

This special issue documents some of the contributed research papers discussed during the proceedings of IUTAM-IITD International Winter School on Optimum Dynamic Design using Modal Testing and Structural Dynamic Modification held at the Indian Institute of Technology, New Delhi from 15th to 19th December 1997. The school was organized with the main objective of providing an opportunity for presenting the latest trends in the area of dynamic design by the internationally known experts in the area. An attempt was made through this school to bring about an awareness of the state-of-art of the software and hardware in various tools of dynamic design, namely dynamic testing, identification, modification and model updating.

Dynamic design aims at obtaining the desired dynamic characteristics in products, equipment, systems and structures by specifying the right shape, size, configuration, material and manufacturing steps of various elements. Desired dynamic characteristics include reduced vibration and noise levels, shifting of natural frequencies or avoidance of resonance, higher dynamic stability and desired mode shapes or vibration patterns. The ultimate objectives are a quieter and more comfortable environment, and higher reliability and quality of product. Dynamic design, which is an area of vibration and noise engineering, has acquired considerable importance due to demands on higher performance capabilities like those of higher speeds, higher power and higher precision of complex mechanical and structural systems like those in transportation, power, machine tools, and other fields. While fulfilling the above mentioned requirements, the designer has to look for solutions within the constraints of economy and a highly evaporative global market. Thus there is a need for lower design and development time which is also taken care of in optimum dynamic design. Further, sound dynamic design of real-life complex systems, like those mentioned above, cannot be ensured on the basis of approaches involving numerical and analytical algorithms. The traditional "cut and try" prototype evaluation and modification procedures to achieve this objective have hitherto been highly time-consuming and expensive.

Research and development in the areas of dynamic testing including modal testing, identification of systems, model updating and structural dynamic modification is most promising for optimal dynamic design. State-of-the-art modal testing methods employing microprocessor-based multichannel FFT analyzers, hammer and multipoint excitation systems, and laser technology have made dynamic test data acquisition quite fast and comprehensive, thus reducing dynamic design time. Compared to earlier traditional testing techniques, this emerges as an optimum dynamic design technology, as dynamic test data have a vital role to play in dynamic design. Computer and dynamic test-based techniques of identification of systems have further optimized the design process by providing the modelled parameters of significance of real systems accurately and quickly. Model updating techniques have helped bring finite element and other analytical dynamic models closer to real systems. Accordingly, the effect of structural modifications on dynamic 
characteristics to improve dynamic design can be predicted at the drawing-board level or more appropriately at the computer level, making the process a highly optimum one in terms of time, energy and money. Further, the dynamic design variables can be optimized for the desired objective functions of cost, weight, power etc. by employing several of the classical optimization algorithms. In view of the above remarks, it is evident that dynamic analysis and therefore development of analytical models coupled with optimization procedures have a very important role to play.

The above mentioned techniques are applicable to simple as well as complex structural and mechanical systems. Information from various sources shows that their successful application to reduce vibrations and improve design covers machine tools, car engines, scooters, tractors, rail vehicles, power generating turbines, aircraft engines and structures, helicopters, satellites and even consumer products like golf clubs, hair clippers, loudspeaker cabinets, lawn mowers, musical instruments, tennis rackets, disk drives of computers, oil sump lines, bicycles and a host of other consumer and industrial products and equipment.

The papers by D J Ewins in this issue cover the basics and state-of-the-art of modal testing, followed by correlation and updating of finite element models, using the results of modal testing so that the approximations inherent in the former can be corrected. The papers by A Sestieri, T K Kundra and B C Nakra deal with structural dynamic modification using various techniques of mass, stiffness and damping modifications in order to achieve the desired dynamic characteristics with the least possible effort. The paper by N Okubo and $\mathrm{T}$ Toi includes sensitivity analysis and its applications for dynamic improvements in mechanical systems. The paper by $\mathrm{R}$ Singh deals with dynamic design of automotive systems including hydraulic engine mounts and structural joints and finally, $\mathrm{N}$ Tandon's paper gives various techniques with applications for noise reducing designs in mechanical and structural systems. It is hoped that the papers included would bring about an awareness of the emerging dynamic design techniques including their principles, applications and the state-of-the-art developments in the area.

We would like to thank Dr G Prathap, Editor, Sädhaña, for his kind invitation and encouragement, and the editorial staff for their interest and effort, in bringing out this special issue.

June 2000

T K KUNDRA

B C NAKRA

Guest Editors 